\title{
A Generalized Diffusion Based Inter-Iteration Nonlinear Bilateral Filtering Scheme for PET Image Reconstruction
}

\author{
Jian Zhou ${ }^{1,2,3,4}$, Hongqing Zhu ${ }^{5}$, Huazhong Shu ${ }^{1,4}$, Limin Luo ${ }^{1,4}$ \\ ${ }^{1}$ Laboratory of Image Science and Technology, Southeast University, Nanjing, China \\ ${ }^{2}$ INSERM U642, France \\ ${ }^{3}$ Universite de Rennes 1, LTSI, Rennes, France \\ ${ }^{4}$ Centre de Recherche en Information Biomedicale Sinograncais (CRIBs), France \\ ${ }^{5}$ East China Univeristy of Science \& Technology, Shanghai, China
}




\section{Abstract}

In this paper, a new inter-iteration filtering scheme based diffusion MAP estimate for PET image reconstruction is proposed. This is achieved by gaining the insights into the classical MAP iteration (e.g. the OSL algorithm) and the several well-established approximations to the diffusion process. We show that such a new technique in turn allows additional insight and sufficient flexibility to further investigate some nonlinear filters based reconstruction algorithms. In particular, upon unraveling the limitations but maintaining the advantages of diffusion regularized method, we suggest the bilateral filter as a nice application in which image smoothing while edge preserving can be readily obtained by the nice combination of the range and domain filters. The feasibility and efficiency of the proposed method are verified in the substantiating experiments conducted on both the computer simulated and real clinical data.

Keywords: image reconstruction, inter-iteration, filtering, MAP, OSL, diffusion, bilateral, range filter, domain filter. 


\section{Introduction}

Positron emission tomography (PET) is one of the most important imaging tools in modern diagnosis; it provides valuable information on the biochemical and biological activity inside a living subject in a non-invasive way. Reconstruction of PET scan images is a complex problem; many algorithms such as analytic and iterative algorithms have been developed in the past decades to solve this problem. Among iterative algorithms, the statistical reconstruction has become increasingly popular due to its ability to model the noise and the imaging physics and to impose the positivity constraints on the reconstruction.

As it is well known, PET is an ill-posed problem; that is, small variations in the data produce large variations in the solution. When using an iterative algorithm, the ML problem in particular, as the likelihood increases, the image starts deteriorating; the algorithm enforces consistency with data, and, because of the size of the problem, more and more high frequencies related to noise show up. So, iterations should be stopped before this happens, by using a suitable stopping criterion [1]. A better approach to overcome the ill-posedness effects is to consider prior information through regularization terms allowing more sophisticated and accurate models.

Bayesian methods known as the Maximum a Posteriori (MAP) estimate for PET image reconstruction and restoration have become increasingly popular because they allow accurate modeling of both data collection and image behavior. The MAP algorithm can remove the divergence in quantitative accuracy at higher iteration numbers, which is often seen in ML due to noise. The result is often a smoother image in the same 
number of iterations. This attributes largely to the introduction of image priori that maintains the reconstruction without being degenerated by the noise. As for the design of priori, the Gibbs distribution is commonly used due greatly to its simplicity and well-established rationale. In the past decades, a variety of prior distributions have been proposed, typically [2], [3], [4], most of which are distinguished by the choice of energy function in the modeling of Gibbs priori. Recently, the diffusion based priori seems especially attractive in case of tomography reconstruction [5]-[8]. It is mainly because the diffusion techniques provide a promising way of image denoising while edge preservation. One may readily construct the diffusion priori by replacing the energy function in the canonical Gibbs distribution with proper gradient based energy functional. Such an insight has led to the inherent deep connections between the MAP estimate and the variational partial differential equations (PDE) based anisotropic diffusion progress [9]. As for the anisotropic diffusion, it has played an important role in signal and image analysis since Perona and Malik's landmark work [10] in which they firstly aimed at persevering sharp features such as edges in images based on nonlinear evolution equation. Their technique may be more simply interpreted as a nonlinear filter whose selective smoothing is based upon the computed local gradient. This promising approach has triggered a tremendous research activity in computer vision and applied mathematics and has been proven to be a powerful tool for restoration and reconstruction of high-quality images.

In this paper, our plan is hence to detail the utilities of nonlinear filtering and help us provide an alternative view of the problem of PET image reconstruction. This, in turn, will be instrumental in our development of an alternative interpretation of existing reconstruction methods, e.g., the 'one-step-late' (OSL) algorithm [3], and in using the gained insight to propose the inter-iteration filtering (IIF) scheme to some of the well 
known limitations. More specifically, we view the reconstruction as a solution to a controlled iteratively prediction/correction system. This ultimately leads to an inter-iteration filtering with two typical filters, the reconstruction filter and the nonlinear filter, well adapted to preserving salient features of images (such as edges) while smoothing away the noise. In doing so, we furthermore introduce the bilateral filter [11] as a nice candidate for the nonlinear filter which enables us to distinguishes noise from signal based on not only the amplitude differences of windowed pixels but also on the spatial size of a detail.

The rest of paper is organized as follows: In section 2, we first briefly outline the problem of PET reconstruction and then introduce the anisotropic diffusion which connects the diffusion based priori and gives us insights to derive accordingly the new reconstruction algorithm based on the IIF scheme; The corresponding evaluations through both the computer simulated and the real clinical data based examples is provided in Section 3. Finally, Section 4 gives out some concluding remarks.

\section{Methodology}

\subsection{PET Image Reconstruction}

In PET, the isotope used emits positrons which annihilate with nearby electrons generating two photons traveling away from each other in opposite direction. The emission of positrons is modeled as a spatial, inhomogeneous Poisson process with unknown intensity, the mean of which is determined by the concentration of the isotope that we wish to estimate. For the convenience of illustration, we assume the 
image to be reconstructed is subdivided into $\sqrt{J} \times \sqrt{J}$ pixels and also assume that the activity concentration within each pixel $j$ is uniform, denoted by $f_{j}$. The data consists of photon coincidence counts collected by $I$ detector pairs, $Y_{i}, i=1,2, \ldots I . Y_{i}$ is a sample from a Poisson distribution whose expectation value is $E\left(\bar{Y}_{i}\right)=[\mathbf{A f}]_{i}=\sum_{j=1}^{J} a_{i j} f_{j}$, where $\bar{Y}_{i}$ is the Poisson random variable corresponding to $Y_{i}$ and the element of the projection matrix $\mathbf{A}=\left\{a_{i j}\right\} \in \mathfrak{R}^{I \times J}, a_{i j}$ is the probability that a positron emitted from pixel $j$ results in a coincidence at the $i$ th LOR, $\mathbf{f}$ is the image vector, i.e., $\mathbf{f}=\left[f_{1}, \cdots, f_{J}\right]^{T}$. The conditional probability of obtaining the measurement vector $\mathbf{Y}$ given the image vector $\mathbf{f}$ (i.e., the likelihood function) is

$$
\operatorname{Pr}(\mathbf{Y} \mid \mathbf{f})=\prod_{i=1}^{I}\left\{\frac{[\mathbf{A} \mathbf{f}]_{i}^{Y_{i}}}{Y_{i} !} \exp \left(-[\mathbf{A} \mathbf{f}]_{i}\right)\right\}
$$

The ML method which estimates $\mathbf{f}$ that maximizes $\operatorname{Pr}(\mathbf{Y} \mid \mathbf{f})$ subject to nonnegative constraints on $\mathbf{f}$, or, equivalently, finds the $\mathbf{f} \geq 0$ which maximizes

$$
l(\mathbf{f})=\sum_{i=1}^{I}\left\{Y_{i} \ln \left([\mathbf{A f}]_{i}\right)-[\mathbf{A f}]_{i}\right\}
$$

With the help of EM algorithm, Shepp and Vardi proposed the following image update formula for the ML solution (ML-EM algorithm) [12]:

$$
\mathbf{f}^{k+1}=\frac{\mathbf{f}^{k}}{\sum_{i=1}^{I} a_{i j}} \sum_{i=1}^{I} \frac{Y_{i} a_{i j}}{\left[\mathbf{A} \mathbf{f}^{k}\right]_{i}}
$$

The ML solution is dominated by noise artifacts, and iterative estimates exhibit increasing noise artifacts along iterations. Therefore regularization techniques are usually needed to produce a reasonable reconstruction. The Bayesian approach as well as the MAP estimate represents a more complete and elegant 
way to stabilize the ML problem by using prior information contained in a regularization term. That is instead of maximizing the likelihood (1), $\operatorname{Pr}(\mathbf{f} \mid \mathbf{Y})$ is maximized, i.e., via the Bayesian formula, the new model becomes

$$
\max _{\mathbf{f} \geq 0} \operatorname{Pr}(\mathbf{f} \mid \mathbf{Y})=\frac{\operatorname{Pr}(\mathbf{Y} \mid \mathbf{f}) \operatorname{Pr}(\mathbf{f})}{\operatorname{Pr}(\mathbf{Y})} \propto \operatorname{Pr}(\mathbf{Y} \mid \mathbf{f}) \operatorname{Pr}(\mathbf{f})
$$

where $P(\mathbf{f})$ and $P(\mathbf{Y})$ are the probabilities of $\mathbf{f}$ and $\mathbf{Y}$ respectively. A common Bayesian priori is the Gibbs distribution of the form:

$$
\operatorname{Pr}(\mathbf{f}) \propto \exp (-\beta \Psi(\mathbf{f})), \quad \beta>0
$$

where $\Psi(\mathbf{f})$ is the so-called energy function. By substituting (5) and (1) into (4) and then taking logarithm, we obtain the MAP estimate:

$$
\hat{\mathbf{f}}=\arg \max _{\mathbf{f} \geq 0}\{l(\mathbf{f})-\beta \Psi(\mathbf{f})\}
$$

One EM-type approach to construct MAP reconstruction $\hat{\mathbf{f}}$ has been recommended by Green [3], which is known as the OSL algorithm. In this algorithm, the influence of prior term is only performed at the current estimate $f^{k}$, and thus results in the simple updating equation [3]:

$$
f_{j}^{k+1}=\frac{f_{j}^{k}}{\sum_{i=1}^{I} a_{i j}+\beta\left(\frac{\partial \Psi(\mathbf{f})}{\left.\partial f_{j}\right|_{\mathbf{f}=\mathbf{f}^{k}}}\right)} \sum_{i=1}^{I} \frac{a_{i j} Y_{i}}{\left[\mathbf{A} \mathbf{f}^{k}\right]_{i}}, j=1, \cdots, J
$$

Apparently such an influence is imposed by the meaning of the gradient of the energy function $U(\mathbf{f})$ evaluated at each iteration $k$. Due to this "one step late" property of this algorithm, a fully iteration can be interestingly seen as an error prediction/correction technique. Calculation of the priori influence, i.e., the gradient of $\Psi(\mathbf{f})$ corresponds to the error prediction, and their use in (7) to reestimate $f_{j}$ is correction. In the proceeding the similar technique will be given new interpretations in our proposed algorithm. 


\subsection{Reconstruction Using Inter-Iteration Filtering Scheme}

\section{A. Anisotropic Diffusion}

In this section, we will discuss the usage of the nonlinear filter for the problem of PET image reconstruction.

It is necessary to review some basic relations between the anisotropic diffusion process and the underlying nonlinear filtering problem. Let us first consider the following energy functional defined on the spaces of smooth images, that is, images for which $\nabla f$ is finite in $\Omega$, as in the following respect to image function $f:$

$$
\Psi(f)=\int_{\Omega} \rho(\|\nabla f\|) d \Omega
$$

where $\rho(\|\nabla f\|) \geq 0$ is an increasing function of $\|\nabla f\|$. One way we can minimize the above expression is by means of gradient descent by using the calculus of variations theory which leads to the parabolic PDE:

$$
\frac{\partial f(x, y, t)}{\partial t}=\Psi^{\prime}=\operatorname{div}\left[\frac{\rho^{\prime}(\|\nabla f\|)}{\|\nabla f\|} \nabla f\right]
$$

with the initial and boundary conditions:

$$
f(x, y, 0)=f^{0} \text {, and }\left.\frac{\partial f}{\partial \vec{N}}\right|_{\partial \Omega}=0
$$

where $\operatorname{div}(\cdot)$ denotes the divergence operator and $\vec{N}$ the outward unit norm of the boundary $\partial \Omega$.

By defining

$$
g(x) \equiv \frac{\rho^{\prime}(x)}{x}
$$

and substituting it into (9) we obtain

$$
\frac{\partial f(x, y, t)}{\partial t}=\operatorname{div}[g(\|\nabla f\|) \nabla f]
$$

which amounts to Perona and Malik's anisotropic diffusion equation [10]. Function $g(\cdot)$ is the so-called 
edge-stopping function that is a nonnegative monotonically decreasing function with $g(0)=1.0$. The diffusion process will mainly take place in the interior of regions, and it will not affect the region boundaries where the magnitude of gradient of $f$ is large. Qualitatively, the effect of anisotropic diffusion is to smooth the original image while preserving brightness discontinuities. This is well controlled by the choice of $g(\cdot)$ that greatly affects the extent to which discontinuities are preserved.

Equation (12) can be discretized as follows [13]:

$$
f_{j}^{k+1}=f_{j}^{k}+\frac{\lambda}{\left|\eta_{j}\right|} g\left(\nabla f_{p, j}\right) \nabla f_{p, j}
$$

where $f_{j}$ is the discretely sampled image, $j$ denotes the pixel position in a discrete 2D grid, and $k$ denotes discrete time steps (iterations). The constant $\lambda \in \mathfrak{R}^{+}$is a scalar that determines the rate of diffusion, $\eta$ represents the spatial neighborhood of pixel $j$, and $\left|\eta_{j}\right|$ is the number of neighbors (usually four). The image gradient is linearly approximated in a particular direction as

$$
\nabla f_{p, j}=f_{p}^{k}-f_{j}^{k}, \quad p \in \eta_{j}
$$

Barash and Comaniciu recently noted that the nonlinear diffusion is common with the generalized adaptive smoothing [14], [15]:

$$
f_{j}^{k+1}=\frac{\sum_{p \in S_{j}} f_{p}^{k} w_{p}}{\sum_{p \in S_{j}} w_{p}}
$$

where the window $S_{j}$ covers a local filter support of size $(2 n+1) \times(2 n+1), n=1,2, \cdots$, centered at the pixel $j$ and $w_{p}$ 's acting as the smoothing kernel correspond to different neighbor combinations with respect to the center pixel of interest. Typically one can choose $w_{p}$ as $w_{p} \equiv g\left(\nabla f_{p, j}\right)$ which results in 
the fundamental link between the anisotropic diffusion and the edge-preserving smooth filters. In the next subsection, we shall give out more details on the design of filter $w_{p}$. It is noted that in practice, $n$ need not be taken too large (usually $n \leq 3$ ), otherwise the generalized adaptive smoothing becomes an inaccurate representation of the extended diffusion equation which causes the image being oversmoothed [15].

\section{B. Inter-Iteration Filtering Scheme}

The diffusion based MAP reconstruction is to consider the continuous prior probability density as follows

[9]:

$$
\operatorname{Pr}(f) \propto \exp \left\{-\beta \int_{\Omega} \rho(\|\nabla f\|) d \Omega\right\}
$$

To approach the corresponding estimate based on the above priori, we can use the insights gaining from the

OSL algorithm that is equivalent to solve the gradient of $\Psi(\mathbf{f})$, or more specifically,

$$
\frac{\partial \Psi(\mathbf{f})}{\partial f_{j}} \cong\left\langle\Psi^{\prime}\right\rangle_{j}
$$

where $\langle F\rangle_{j}$ denotes the $j$ th sample of continuous function $F$. One may reach (17) by properly discretizing $\Psi^{\prime}$ as well as the divergence operator in (2), which usually involves the finite difference to the first and second order partial derivatives and possibly becomes complicated with the different choices of $\rho(\cdot)$. However, by noticing the relations between the anisotropic diffusion (13) and the generalized adaptive smoothing (15), we may instead approximate (17) by using the following slightly abusive form

$$
\left.\left.\frac{\partial \Psi(\mathbf{f})}{\partial f_{j}}\right|_{\mathbf{f}=\mathbf{f}^{k}} \cong\left\{\left(\frac{\sum_{p \in S_{j}} f_{p} w_{p}}{\sum_{p \in S_{j}} w_{p}}\right)-f_{j}\right\}\right|_{\mathbf{f}=\mathbf{f}^{k}} .
$$

Clearly such an approximation is easier to manipulate since it depends only on the choice of smoothing 
kernel $w_{p}$. Secondly, perhaps the most importantly, because the first term on the right-hand side of (18) acts like the nonlinear filtering carried on a local structure $S_{j}$ of the current estimate $\mathbf{f}^{k}$, it thus can be treated independently. Therefore, it is worthy to note that (18) does indicate how the reconstruction can be reinterpreted as an inter-iteration filtering scheme.

More precisely, by substituting (18) into the OSL formula (7), we can present the new algorithm as follows:

$$
f_{j}^{k+1}=\frac{f_{j}^{k}}{\sum_{i=1}^{I} a_{i j}+\beta\left(\left[\mathbf{H}^{k} \mathbf{f}^{k}\right]_{j}-f_{j}^{k}\right)} \sum_{i=1}^{I} \frac{a_{i j} Y_{i}}{\left[\mathbf{A} \mathbf{f}^{k}\right]_{i}}, \quad \text { for each } j=1, \cdots, J
$$

where $\mathbf{H}^{k}$ represents the edge-preserving smooth filter at iteration $k$ and for each $j=1, \cdots, J$ we typically have

$$
\left[\mathbf{H}^{k} \mathbf{f}^{k}\right]_{j}=\frac{\sum_{p \in S_{j}} f_{p}^{k} w_{p}}{\sum_{p \in S_{j}} w_{p}}
$$

The above iteration scheme furthermore can be summarized in Fig.1.

\section{FIGURE 1}

In Fig.1, we describe the reconstruction in the notation of filtering which is also in the spirits of OSL's prediction/correction technique. Given current estimate $\mathbf{f}^{k}$, the $\tilde{\mathbf{f}}^{k}$ is obtained by inputting $\mathbf{f}^{k}$ to the nonlinear filter $\mathbf{H}^{k}$. The output is then subtracted by $\mathbf{f}^{k}$ to produce the residue error $\mathbf{e}^{k}$ which along with the $\mathbf{f}^{k}$ and observation $\mathbf{Y}$ feeds back to the reconstruction filter to yield the new estimate $\mathbf{f}^{k+1}$. 


\section{Bilateral Filter}

Comparing most other diffusion based MAP algorithms, e.g., [6]-[8], the proposed algorithm treats the diffusion based a priori more implicitly so that we can focus only on the design of smooth filter $\mathbf{H}^{k}$ as well as the weights $w_{p}$ 's. As mentioned previously, $w_{p}$ can be taken as $g\left(\nabla f_{p, j}\right)$ and then lead to the common diffusion filter. However such a filter usually does not consider the pixel position information but emphasize only on their differences to the center pixel of the specific local structure $S_{j}$. Thus the effects of spatial resolution are often ignored and can not be well maintained for the case of practical uses.

One way out of this problem is to model the spatial resolution together with the mentioned diffusion filter which results in the bilateral filter. The bilateral filtering was first introduced in [11] as the nonlinear filter which combines domain and range filtering. It has proven to be useful in the accomplishment of compute vision tasks [16], [17] and its nice properties have been well established in several literatures, such as [11], [18], [19], [20]. Given an input image $f$, using a continuous representation notation as in [11], the output image $\tilde{f}$ is obtained by

$$
\tilde{f}(\mathbf{x})=\frac{\int_{-\infty}^{+\infty} \int_{-\infty}^{+\infty} f(\mathbf{x}) c(\xi, \mathbf{x}) s(f(\xi), f(\mathbf{x})) d \xi}{\int_{-\infty}^{+\infty} \int_{-\infty}^{+\infty} c(\xi, \mathbf{x}) s(f(\xi), f(\mathbf{x})) d \xi}
$$

where $\mathbf{x}=(x, y), \xi=\left(\xi_{1}, \xi_{2}\right)$ are space variables. Clearly the convolution kernel is the product of the functions $c$ and $s$, which represent 'closeness' (in the domain) and 'similarity' (in the range), respectively. In case of discrete image, each weight within the specific window $S_{j}$ then can be determined by

$$
w_{p}=c(p, j) s\left(f_{p}, f_{j}\right) .
$$


More specifically, $c(\cdot \cdot)$ is usually radially symmetric, taking the Gaussian like function for example [11],

$$
c(p, j)=\exp \left(-\frac{d(p, j)^{2}}{2 \sigma_{D}^{2}}\right)
$$

where

$$
d(p, j)=d(p-j)=\|p-j\|
$$

is the Euclidean distance between pixel $p$ and $j, \sigma_{D}$ denotes the geometric spread controlling the shape of Gaussian function. In such a case, we found that the function $c(\cdot$,$) acts more like the point spread$ function (PSF) of a typical imaging system. Therefore, $\sigma_{D}$ can be similarly characterized with the system spatial resolution by meaning of full-width-at-half-maximum (FWHM).

The similarity function $s(\cdot, \cdot)$ employed here is defined by

$$
s\left(f_{p}, f_{j}\right)=\exp \left(-\frac{\left|f_{p}-f_{j}\right|}{\sigma_{R}^{2}}\right)
$$

where $\sigma_{R}$ is the tuning parameter. The similarity function in (25) acts like a Laplacian distribution and is bounded above by unity. Consequently, the function $s(\cdot, \cdot)$ maintaining the properties of the edge-stopping function $g(\cdot)\left(\right.$ i.e., $\left.s\left(f_{p}, f_{j}\right) \equiv g\left(\nabla f_{p, j}\right)\right)$ progressively penalizes intensity differences, and thus favors locally smooth images, but by controlling the behavior for large intensity differences through $\sigma_{R}$, true discontinuities in the image are not overpenalized. We have found in experiments, with an appropriate choice of $\sigma_{R}$, It can yield reconstructions which are locally smooth but do not appear to oversmooth across intensity boundaries.

Towards the end, we refer to the proposed algorithm as IIF-MAP and outline it as follows: 
1) Setup the initial guess $\mathbf{f}^{0}$ ( $\mathbf{f}^{0} \geq 0$, e.g., FBP reconstruction); Select constant values for parameter $\beta$ and $\sigma_{R}$; Determine the size of window $n$ then $\sigma_{D}$. For $k=0,1,2, \cdots$, we start with the following steps:

2) Construct the bilateral filter $\mathbf{H}^{k}$ using (22)-(25) combining with the current estimate $\mathbf{f}^{k}$;

3) Filter $\mathbf{f}^{k}$ using (20) to obtain the $\tilde{\mathbf{f}}^{k}$ and then compute the error prediction $\mathbf{e}^{k}=\tilde{\mathbf{f}}^{k}-\mathbf{f}^{k}$;

4) Reestimate the new reconstruction $\mathbf{f}^{k+1}$ using (19);

5) Check whether iteration reaches some specific stopping conditions. If no, return to step 2) and repeat again; otherwise, quit the loop of iteration and output the reconstruction.

\section{Experimental Results and Discussions}

This section presents reconstructions of simulated and clinical data using the ML-EM algorithm and the IIF-MAP algorithm using the nonlinear bilateral filter introduced in section 2. PET emission data was simulated using the computer generated brain phantom shown in figure 2(a). The phantom is of size $128 \times 128$ pixels. The grey levels in figure 2 (a) reflect the relative activities in different regions of the phantom. Three types of matter: grey matter, white matter and CSF were used, which were then assigned to be $5.64,2.46$, and 1.0 respectively [21]. The sinogram was generated by forward projection of the brain phantom image. Simulated Poisson noise equivalent of one million counts was added to the projections. Complicating factors such as attenuation and scatter were not considered. The image was projected in 192 equiangular directions onto an array of 192 equispaced coincidence detector pairs per angle, resulting in a sinogram of size $192 \times 192$. 


\subsection{ML-EM Reconstruction of simulated data}

The brain phantom sinogram was reconstructed using the ML-EM algorithm given by equation (3). The initial image $\mathbf{f}^{0}$ was taken to be uniform with a total of approximately one million counts. One hundred iterations of the ML-EM algorithm were performed. The reconstructions of the brain phantom at iterations 10, 20, 50, and 100 are shown in figure 2(b)-(e). The gradual increase in noise with the number of iterations is apparent in these images. The deterioration in image quality can be illustrated by computing the normalized mean square error (MSE) between the simulated noise-free activity distribution and the image estimate as a function of the iteration number $k$. The MSE is given by

$$
\operatorname{MSE}(k)=\frac{\left\|\mathbf{f}^{k}-\mathbf{f}^{\text {true }}\right\|^{2}}{\left\|\mathbf{f}^{\text {true }}\right\|^{2}} \times 100 \%
$$

where $\mathbf{f}^{\text {true }}$ denotes the ground-truth phantom. The MSE for the ML-EM reconstruction of the brain phantom is shown in figure 4. For this particular study, the minimum MSE was reached at iteration number 34, after which the MSE began to increase. As expected, the log-likelihood function (2) kept increasing with the iteration number while the image quality deteriorated [1]. 


\subsection{IIF-MAP Reconstruction of simulated data}

IIF-MAP reconstructions of simulated data are presented in this section and the results are compared with

ML-EM and other MAP reconstruction. As in the case of ML-EM, the initial image estimate $\mathbf{f}^{0}$ was also chosen to be uniform with a total of approximately one million counts. Another MAP used here is with the priori of total variational (TV) in which the function $\rho(\|\nabla f\|)$ is defined by:

$$
\rho(\|\nabla f\|)=\|\nabla f\|
$$

It is known (e.g., [22]) that TV based diffusion process relates closely to the so-called mean curvature flow that has proven to be powerful for image smoothing while edge preserving. In this paper, the resulting MAP reconstruction method is named as TV-MAP. According to (9), the gradient is

$$
\Psi^{\prime}=\operatorname{div}\left[\frac{\nabla f}{\|\nabla f\|}\right] .
$$

With some knowledge of algebra, the right-hand side of above formula can be written out analytically [22]:

$$
\operatorname{div}\left[\frac{\nabla f}{\|\nabla f\|}\right] \equiv \frac{f_{x x} f_{y}^{2}-2 f_{x} f_{y} f_{x y}+f_{y y} f_{x}^{2}}{\left(\sqrt{f_{x}^{2}+f_{y}^{2}}\right)^{3}}
$$

where $f_{x}$ and $f_{y}$ are the first-order partial derivatives of function $f$ with respect to the coordinate $x$ and $y ; f_{x x}, f_{y y}, f_{x y}$ are the related second-order partial derivatives. In order to well approximate each partial derivative, the scheme of finite difference can be used. For the convenience of illustration, we introduce $(x, y)(x=1,2, \ldots, \sqrt{J}$ and $y=1,2, \ldots, \sqrt{J})$ to index pixel location of any two-dimensional function, and also suppose the difference step is unity. With this help, the derivative approximation can be yielded by:

1) The first-order derivatives:

$$
f_{x}(x, y) \cong[f(x+1, y)-f(x-1, y)] / 2, \quad f_{y}(x, y) \cong[f(x, y+1)-f(x, y-1)] / 2 ;
$$


2) The second-order derivatives:

$$
\begin{gathered}
f_{x x} \cong f(x+1, y)-2 f(x, y)+f(x-1, y), f_{y y} \cong f(x, y+1)-2 f(x, y)+f(x, y-1), \\
f_{x y} \cong[f(x+1, y+1)+f(x-1, y-1)-f(x+1, y-1)-f(x-1, y+1)] / 4 .
\end{gathered}
$$

Here we can use either the zero-padding or boundary reduplication to avoid the error of boundary violation.

By using the coordinate transform, i.e., $\Psi^{\prime}(x, y) \equiv\left\langle\Psi^{\prime}\right\rangle_{(x-1) \sqrt{M}+y}$, we can obtain the required vector version of gradient. Also it is worthy noting that, to stabilize the gradient calculation, $\sqrt{f_{x}^{2}+f_{y}^{2}}$ in the denominator of (27) has been replaced with $\sqrt{f_{x}^{2}+f_{y}^{2}+\varepsilon}$ (where $\varepsilon=10^{-5}$ ) in order to avoid the divide by zero [23].

In the IIF-MAP algorithm, we apply the bilateral filter to penalize the noise artefact. In the bilateral filter, the closeness function as well as the domain filter is determined by (23) whose shape is mainly controlled by the variance of Gaussian function $\sigma_{D}$. In this paper, we choose $\sigma_{D}$ as the function of window size $n$ and the tuning parameter $\gamma$,

$$
\sigma_{D}=\sqrt{-\frac{2 n^{2}}{\ln \gamma}}, \quad 0<\gamma<1.0
$$

Here $\gamma$ is the ratio to the peak of Gaussian window function. Apparently, the smaller the $\gamma$ is, the larger the $\sigma_{D}$ as well as the smaller the influences that pixels far from the center to the center pixel of interest will be. In this paper we select $\gamma=50 \%$ so that the $\gamma$ has the same effect as the FWHM of the system. Therefore, the resultant $\sigma_{D}$ 's for windows with size of $3 \times 3,5 \times 5$ and $7 \times 7$ are $1.6986,3.3973,5.0959$ respectively. 
Firstly we investigate the influence of regularization parameter $\beta$ in our IIF-MAP. Reconstruction for three different values of $\beta$ with $\sigma_{R}=0.2$ and $n=1$ is shown in figure 3 .

\section{FIGURE 3}

As is evidenced in this figure, IIF-MAP reconstruction with the bilateral filter greatly alleviates the noise artifacts in the reconstructed image. It can be seen that $\beta$ controls the degree of smoothness of the IIF-MAP reconstruction. A striking improvement can be found out that although the reconstructed images tend to be smooth, edges can be well preserved (see figure 3(b)) by choosing appropriate value $\beta$. This is mainly due to the used bilateral filter which smoothes the noise while persevere salient features as well. As the additional evidence, figure 4 shows the related line comparisons between different reconstructions where the edge-preserving smoothing property of IIF-MAP can be readily found out.

FIGURE 4

\section{FIGURE 5}

Figure 5 compares the MSE between the ML-EM and the IIF-MAP reconstructions as a function of iteration number. As noted early, the MSE in ML-EM reconstruction reaches its minimum at iteration number 34 and then starts to increase with increasing of iteration number. However, the MSE in the 
IIF-MAP algorithm continues to decrease. It also indicates from the IIF-MAP reconstructions shown in figure 3 that the smoothing achieved by the IIF-MAP algorithm with inter-iteration filtering results in a loss of resolution in the reconstructed images. This effect has been also observed in many other MAP image reconstructions (e.g., [2], [3]). In fact, the IIF-MAP method can easily be reformulated as a Gaussian like MAP estimate by choosing the priori probability density as follows:

$$
\operatorname{Pr}(\mathbf{f}) \propto \exp \left[-\beta \sum_{j=1}^{J}\left(f_{j}-\mu_{j}\right)^{2}\right]
$$

where $\mu_{j}$ denote the mean Gaussian variables. Clearly we can set

$$
\mu_{j}=\left[\mathbf{H}^{k} \mathbf{f}\right]_{j}
$$

and then readily obtain the same expression as (19).

The performance of IIF-MAP was further addressed by comparing it with TV-MAP. For each MAP algorithm, we started with the same uniform image and then perform 100 iterations. To obtain the relatively optimal value of $\beta$ for each MAP, a greedy strategy was used, which produced 100 different $\beta$ values uniformly spaced in the specific interval $[0.001,0.50]$. The minimum of MSE for each $\beta$ was saved throughout the iteration progress, which is finally plotted as the function $\beta$ in figure 6(a).

\section{FIGURE 6}

It is clear to see that the optimal $\beta$ varies with the used MAP method. In this particular experiment, $\beta=0.006$ yields the global MSE minimum (MSE $=6.7716)$ in TV-MAP while $\beta=0.102$ seems 
optimal for IIF-MAP (MSE = 6.1854). Figure 6(b) shows the reconstructed images that correspond to the above two cases, i.e., $\beta=0.006$ for TV-MAP and $\beta=0.102$ for IIF-MAP. As we can see, both of images are quite similar at least from the visual standard of view, except for the TV-MAP reconstruction which displays several "black dots" artefact that, we believe, are caused by the numerical instability during the calculation of the required gradient operation. Actually, one may alleviate this problem by increasing the value of $\varepsilon$. However we note here too large $\varepsilon$ does change the property of TV that may result in a totally different method. As additional evidence, figure 6(c) shows the related line comparisons between different reconstructions of TV-MAP and IIF-MAP in which the advantage of IIF-MAP can be viewed.

For each MAP algorithm, we also recorded the execute time calculated by averaging those CPU time costs over 100 iteration. Figure 7 shows the time cost curves generated by using TV-MAP and IIF-MAP, where labels indicate the average cost for each 5 iteration. The total average here is 1.2202 s for TV-MAP and 0.7385s for IIF-MAP respectively. It is easy to view that IIF-MAP needs few time than TV-MAP, and nearly $40 \%$ cost is reduced. This is because the computation of bilateral filtering can be relatively more convenient than that of TV diffusion term (27).

\section{FIGURE 7}

We shall note here that the results are provided just in our particular experimental context, which in usual not only depend on the algorithm itself, but also can change with the programming language, code optimization and even the configuration of the used computer. In our experiment, all of programs are coded 
in $\mathrm{C}++$. The computer used for simulation is with the configuration: 2.4GHz CPU and 1.0GBytes memory storage.

We also study the effects of parameter $\sigma_{R}$ and $n$ by fixing the parameter $\beta$ at 0.25 . Reconstructions using three different values of $\sigma_{R}$ and various sizes of window $n$ are shown in figure 8 . Here smooth images are achieved by either large $n$ or $\sigma_{R}$, as which is indicated in section 2. Similarly, smooth regions with sharp boundary edges can also be well preserved by relatively small value of $\sigma_{R}$. For this particular experiment, $\sigma_{R}=0.2$ and $n=1$ seem to give out the best reconstruction. However, it is worthy to note that too small $\sigma_{R}$ does not offer promising reconstructions as we can view from the first row of figure $8\left(\sigma_{R}=0.05\right)$. In such a case, pixels with only small difference to the center one would be overweighed so that numerous fault edges will be erroneously preserved and cause instead the noisy reconstruction. As indicated previously, given the similarity function (28) used in the bilateral filter, $\sigma_{R}$ acts as an average edge threshold and the choice of which largely depends on the noise characteristics of measurement data. There are lots of approaches to the optimal choice of $\sigma_{R}$. For example, one can estimate $\sigma_{R}$ by either using the prior knowledge, such as the FBP reconstruction, or adaptively calculating it via the robust estimate method as it was recommended by Black et al [13]. Detailed discussion can be found in literature [5]. We shall refer the interested reader to these literatures for more details.

\section{FIGURE 8}




\subsection{Reconstruction of clinical data}

This section deals with the reconstruction for the real clinical brain data. PET raw data were measured by the currently state-of-art multi-ring PET/CT scanner (CTI Siemens Biograph Sensation 16). It can provide the highest transaxial resolution of $6.2 \mathrm{~mm}$ FWHM and axial resolution of $4.3 \mathrm{~mm}$ FWHM in case of two-dimensional imaging [24]. The data acquisition commenced with the arrival of the radioactivity in the human brain and lasted for about 30 minutes. A total number of 47 image planes were generated and the emission data for each plane were stored in term of sinogram with size $192 \times 192$. The reconstructed images were all set to the $128 \times 128$ pixel matrix with pixel size $2.0 \mathrm{~mm}$. Similarly, normalization of detector efficiencies was performed using measurements obtained from a rotating line source prior to image reconstruction. Random coincidences were measured using the delayed time window method. The random coincidences measured in every detector pair were subtracted from the total number of coincidences detected by that pair.

Reconstructions for several selected image planes are shown in figure 9. The reconstructions were obtained by starting from a uniform initial image. The characteristics of the reconstructed images confirmed the findings based on simulation studies. Images in the first row of figure 9 were obtained by running 100 iterations of the ML-EM algorithm. The ML-EM reconstruction was characteristically noisy. However, the bias in the ML-EM reconstruction decreases with the number of iterations even though the noise is increasing. For the using of IIF-MAP algorithm, we chose $n$ to be 2 so that the resulting resolution can be appropriate to the above given system FHWM. Images shown in the second and third row of figure 9 were 
obtained by running 100 iterations of TV-MAP and IIF-MAP respectively. Both MAP algorithms allow the continuation of the iterations without the noise artifact but TV-MAP is slightly inferior to IIF-MAP by producing several artefacts as demonstrated in the previous subsection. Because of the same size of used measurement data and reconstruction, the time costs when using these MAP methods were similar to the results as shown in the computer simulated experiments. Here TV-MAP needs 1.2326s while IIF-MAP only 0.7322s for every iteration.

\section{FIGURE 9}

In addition to these reconstructions using MAP, the regularization parameter $\beta$ is chosen arbitrary since it has proved to be difficult in clinical routine, due to the fact that a given regularization weight produces different smoothing levels according to the patient dependent model. However, there exist a number of methods in the literature for estimating the regularization parameter in image restoration and reconstruction problems. A number of alternative approaches for selecting regularization parameters have also been early studied in [25], [26]. Presently, it is not clear which one of these techniques is most suitable for the present problem of clinical PET image reconstruction. Future work is needed to study the application of these and other methods to the present problem in order to determine the optimal method for selecting $\beta$. Finally, we do not provide any proof of the convergence of the IIF-MAP algorithm, as this will depend on the form of the used filter and even the value of $\beta$. However, empirical evidence suggests that it usually converges when the filter is as defined in section 2 and $\beta$ is chosen properly. 


\section{Conclusion}

To summarize, we have proposed the new method for PET image reconstruction. In this method, the commonly used diffusion based a priori regularization is naturally approached by the new generalized inter-iteration filtering scheme. Furthermore, to overcome the filtering task but pertain to the diffusion based MAP estimate, the extension nonlinear filter as well as the bilateral filter was employed, which preserves the salient features of reconstructed image while smoothes away the noise. The qualitative and quantitative evaluations of the reconstructions presented in this paper clearly indicated the feasibility and efficiency in the reconstructed images when the IIF coupled with the bilateral filter is used. 


\section{Reference}

[1] J. Llacer J and E. Veklerov, "Feasible images and practical stopping rules for iterative algorithms in emission tomography," IEEE Trans. Med. Imag., vol. 8, pp. 186-93, 1989.

[2] T. Hebert and R. Leahy, "A generalized EM algorithm for 3-d Bayesian reconstruction from Poisson data using Gibbs priors," IEEE Trans. Med. Imag., vol. 8, pp. 194-202, 1989.

[3] P. J. Green, "Bayesian reconstructions from emission tomography data using a modified EM algorithm," IEEE Trans. Med. Imag., vol. 9, pp. 84-93, 1990.

[4] D. S. Lalush and B. M. W. Tsui, "A generalized Gibbs prior for maximum a posteriori reconstruction in SPECT," Phys. Med. Biol. vol. 38 pp. 729-41, 1993.

[5] F. J. Beekman, E. T. P. Slijpen and W. J. Niessen, "Selection of task-dependent diffusion filters for the post-processing of SPECT images," Phys. Med. Biol., vol. 43, pp. 1713-1730, 1998.

[6] O. Demirkaya, "Improving SNR in PET images by using anisotropic diffusion filtration," Proceeding of the $22^{\text {nd }}$ Annual EMBS International Conference, July 23-28, Chicago IL. 2000.

[7] C. Riddell, I. Buvat, A. Savi, M. C. Gilardi, and F. Fazio, "Iterative reconstruction of SPECT data with adaptive regularization,” IEEE Trans. Nucl. Sci., vol. 49, pp. 2350-2354, 2002.

[8] C. Riddell, H. Benali and I. Buvat, "Diffusion Regularization for Iterative Reconstruction in Emission tomography," IEEE Trans. Nucl. Sci., vol. 51, pp. 712-718, 2004.

[9] A. B. Hamza, H. Krim and G. B. Unal, "Unifying probabilistic and variational estimation," IEEE Signal Processing Magazine, vol. 19, pp. 37-47, 2002.

[10] P. Perona and J. Malik, "Scale-Space and Edge Detection Using Anisotropic Diffusion," IEEE Trans. 
Pattern Analysis and Machine Intelligence, vol. 12, no. 7, pp. 629-639, July 1990.

[11] C. Tomasi, R. Manduchi, "Bilateral filtering for gray and color images," Proceedings of the 1998 IEEE International Conference on Computer Vision, Bombay, India, 1998.

[12] L. A. Shepp and Y. Vardi, "Maximum likelihood reconstruction for emission tomography," IEEE Trans. Med. Imag. vol. 1, pp. 113-122, 1982.

[13] M.J. Black, G. Sapiro, D.H. Marimont, and D. Heeger, "Robust Anisotropic Diffusion," IEEE Trans. Image Processing, vol. 7, no. 3, pp. 421-432, 1998.

[14] D. Barash, "Bilateral filtering and anisotropic diffusion: towards a unified viewpoint," Hewlett-Packard Laboratories Technical Report, HPL-2000-18(R.1), 2000.

[15] D. Barash, D. Comaniciu, "A common framework for nonlinear diffusion, adaptive smoothing, bilateral filtering and mean shift," Image and Vision Computing, vol. 22, pp. 73-81, 2003.

[16] N. Sochen, R. Kimmel and A.M. Bruckstein, "Diffusions and confusions in signal and image processing," Journal of Mathematical Imaging and Vision, vol. 14, pp. 195-207, 2001.

[17] T.F. Chan, S. Osher and J. Shen, "The digital TV filter and nonlinear denoising," IEEE Trans. Imag. Proc., vol.10, pp. 231-241, 2001.

[18] R. Kimmel, R. Malladi and N. Sochen, "Images as embedding maps and minimal surfaces: movies, color, and volumetric medical images," Proceedings of the IEEE Computer Society Conference on Computer Vision and Pattern Recognition, Puerto Rico, 1997.

[19] M. Elad, On the bilateral filter and ways to improve it, IEEE Trans. Imag. Proc. vol. 11, pp. 1141-1151, 2002.

[20] N. Sochen, R. Kimmel and R. Malladi, A general framework for low level vision, IEEE Trans. Imag. 
Proc. vol. 7, pp. 310-318 1998.

[21] B. A. Ardekani, et al, "Minimum cross-entropy reconstruction of PET images using prior anatomical information," Phys. Med. Biol. vol. 41, pp. 2497-2517, 1996.

[22] G. Sapiro, "Geometric Partial Differential Equations And Image Analysis," Cambridge University Press. 2001.

[23] C. R. Vogel and M. E. Oman. "Iterative methods for total variation denoising," SIAM J. Sci. Statist. Comput., vol. 17, pp. 227-238, 1996.

[24] G. Tarantola, F. Zito and P. Gerundini, "PET instrumentation and reconstruction algorithm in whole-body applications," J. of Nuclear Medicine, vol. 44, pp. 756-769, 2003.

[25] A. M. Thompson, J. C. Brown, J. W. Kay and D. M. Titterington, "A study of methods of choosing the smoothing parameter in image restoration by regularization," IEEE Trans. Pattern Anal. Machine Intell. vol. 13, pp. 326-38, 1991.

[26] R. Molina, "On the hierarchical Bayesian approach to image restoration: Applications to astronomical images," IEEE Trans. Pattern Anal. Machine Intell. vol. 16, pp. 1122-8, 1994. 


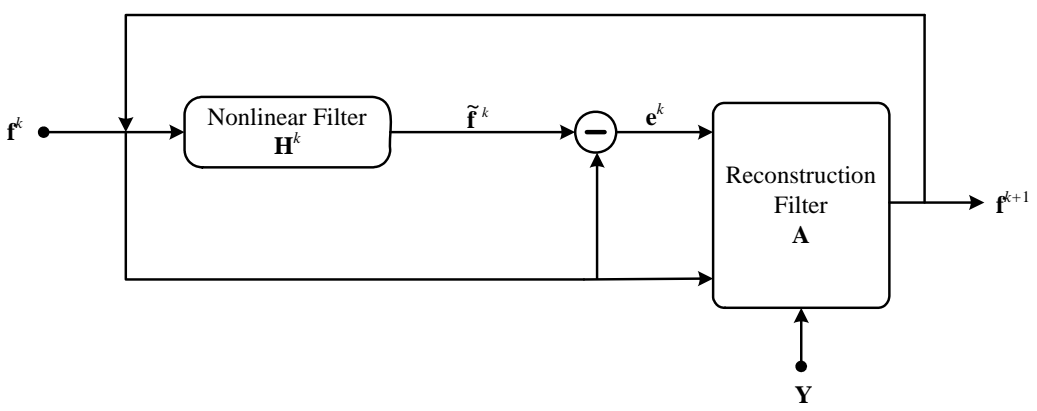

Figure 1: The inter-iteration filtering scheme

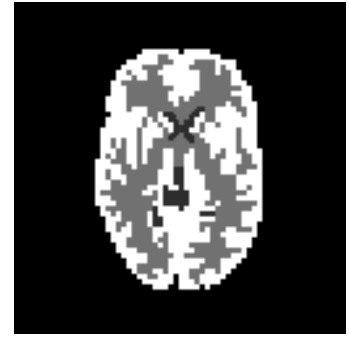

(a)

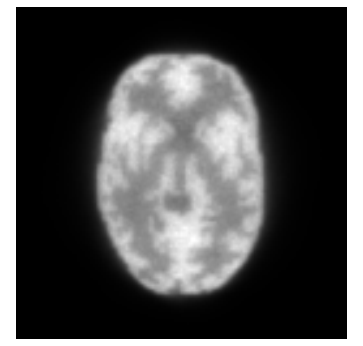

(b)

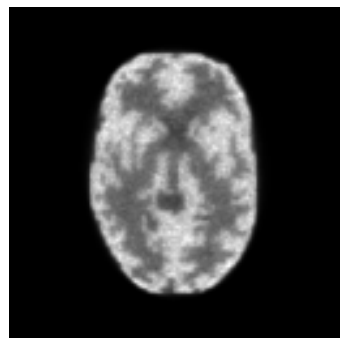

(c)

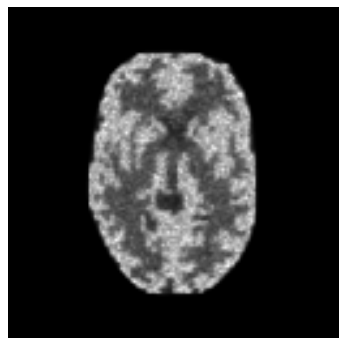

(d)

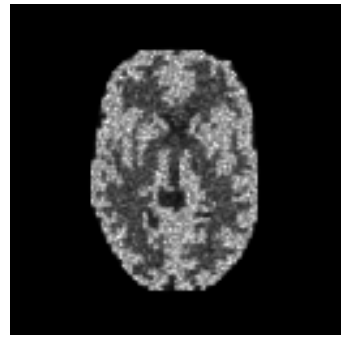

(e)

Figure 2: (a) the Hoffman brain phantom, (b)-(e) ML-EM reconstructions of the simulated emission data at iterations 10, 20, 50 and 100. 


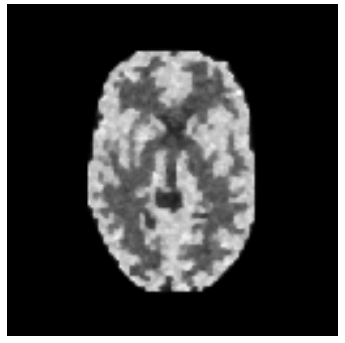

(a)

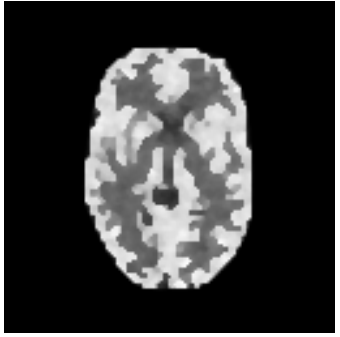

(b)

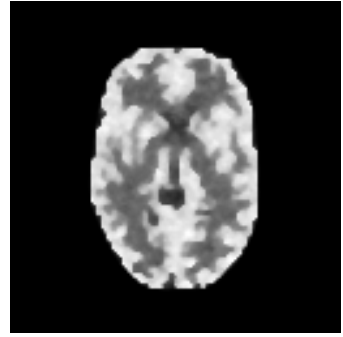

(c)

Figure 3: Reconstructions of the brain phantom obtained by running 100 iterations of the IIF-MAP algorithm using the bilateral filter with (a) $\beta=0.15$ (b) $\beta=0.25$ (c) $\beta=0.35$. ( $\sigma_{R}=0.2$ and $n=1$ for all the case)

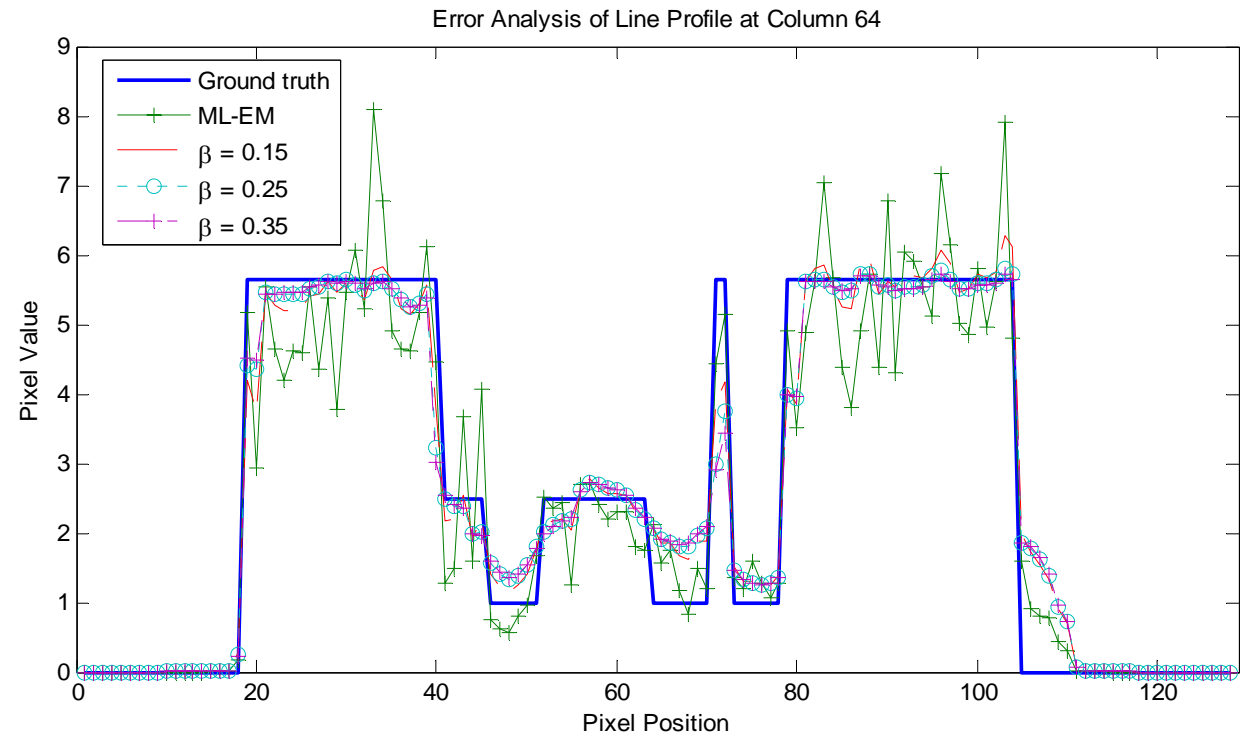

Figure 4: Comparisons of line profile at column 64 of reconstructed images. 


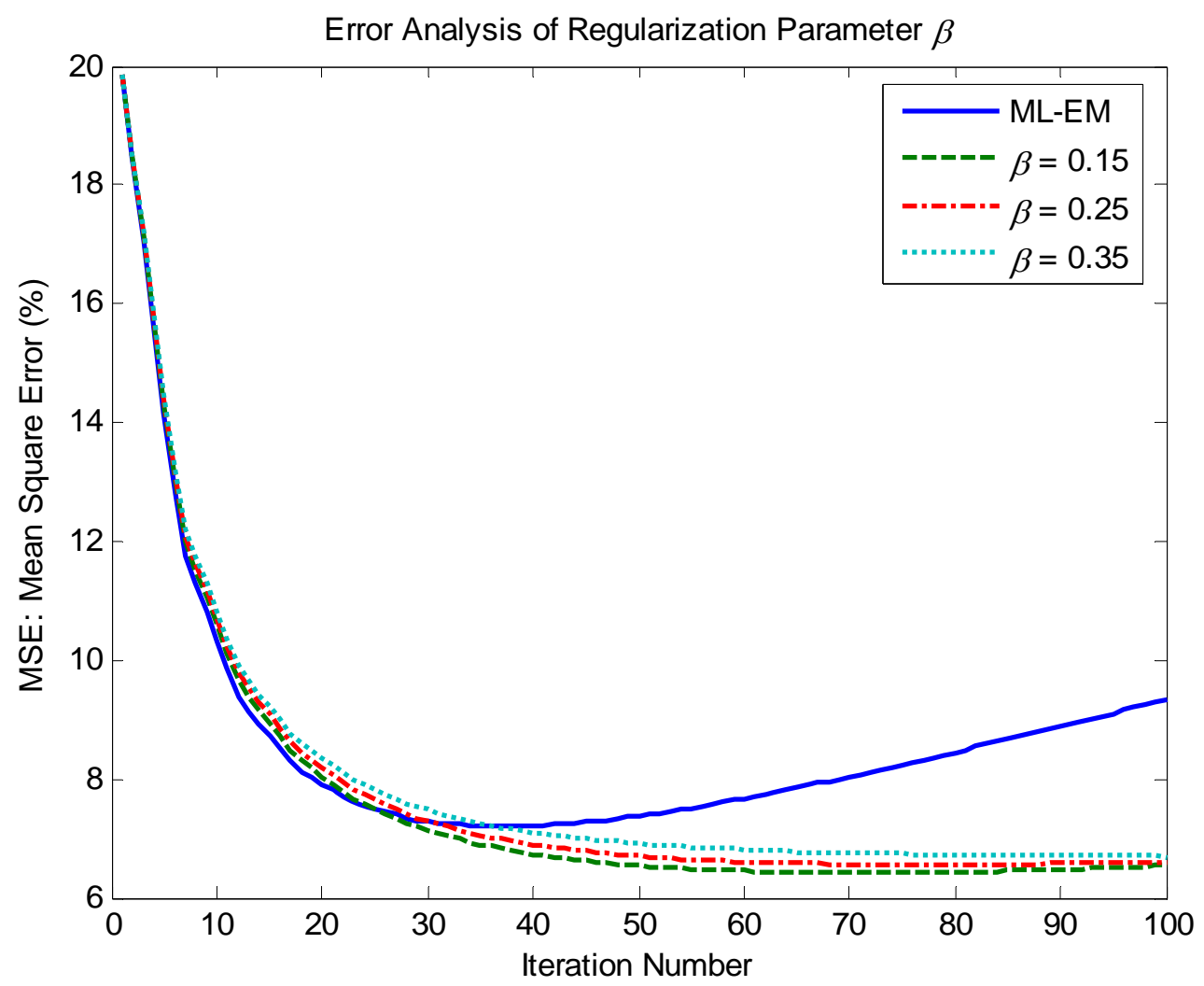

Figure 5: The MSE between the simulated noise-free phantom image and the ML-EM and IIF-MAP (using different regularization parameter $\beta$ ) reconstructed images as a function of iteration number. 


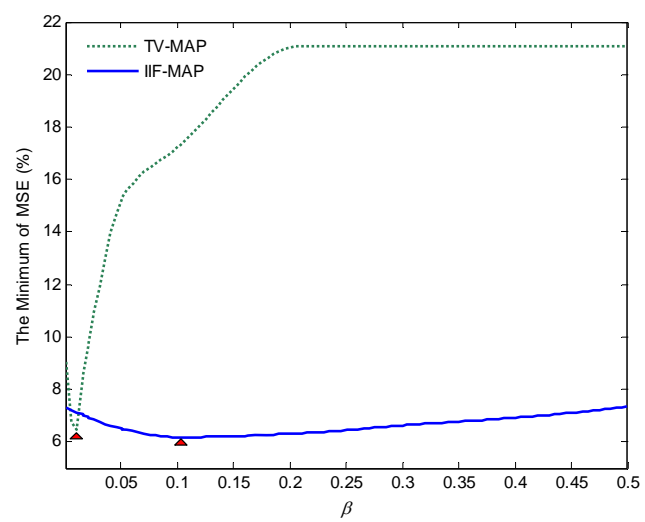

(a)

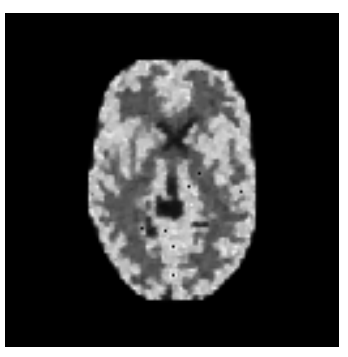

(b)

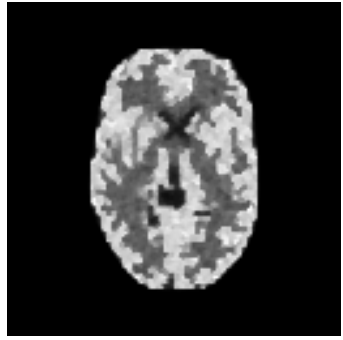

(c)

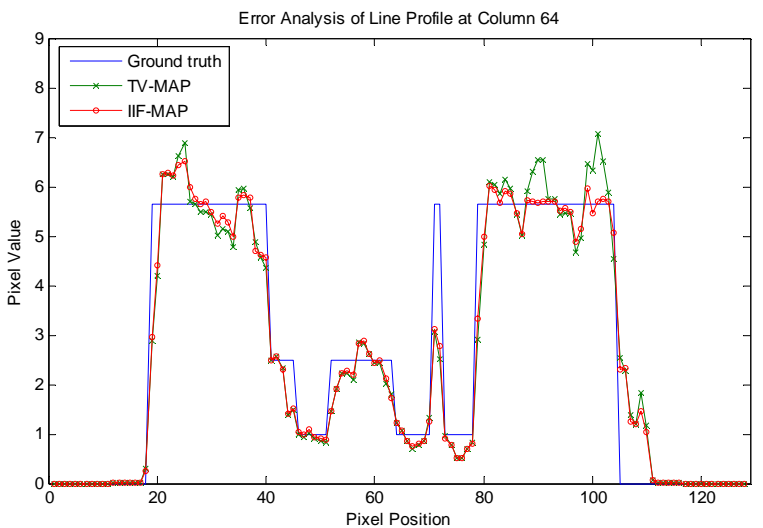

(d)

Figure 6: (a) The minimum MSE changes as a function of $\beta$ where the triangles locate the global minimum MSE as well the optimal $\beta$ for different MAP method; (b) and (c) are reconstructions using TV-MAP and IIF-MAP ( $\left.n=1, \sigma_{R}=0.2\right)$ respectively with optimal $\beta$ indicated in (a); (d) The comparison of line profile of images shown in (b) (c). 


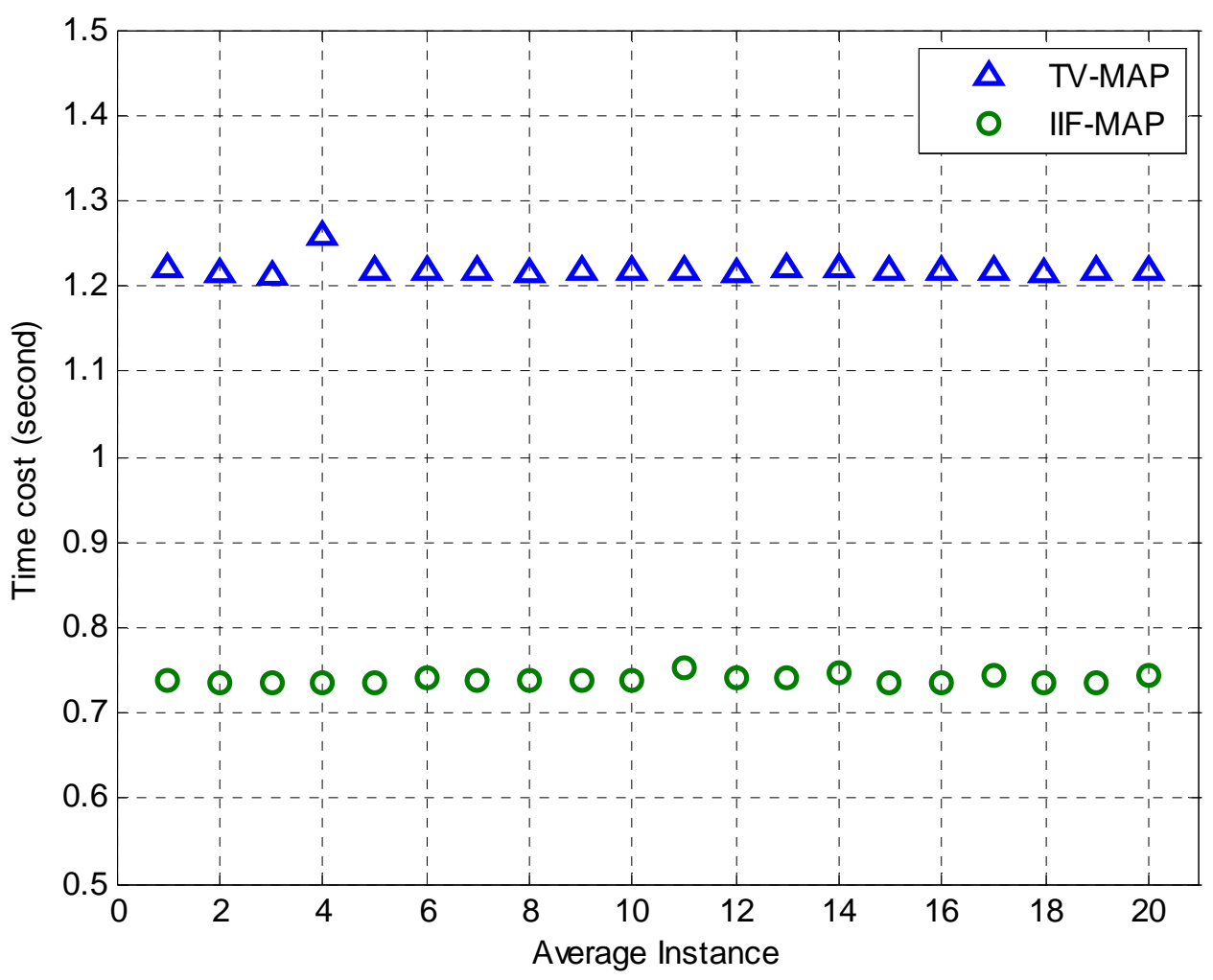

Figure 7: Time cost curves generated by using TV-MAP and IIF-MAP. 
$\sigma_{R}$

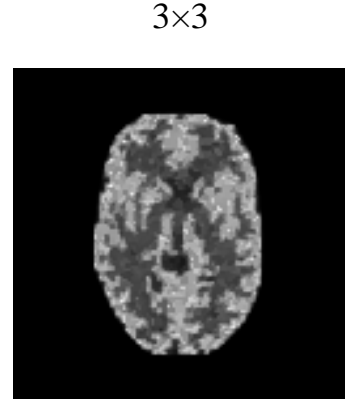

0.2

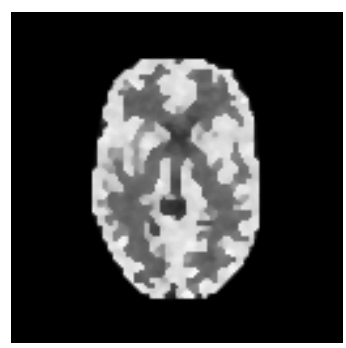

0.4

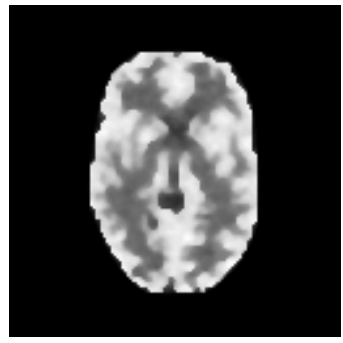

0.8

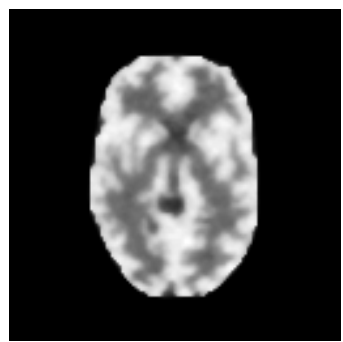

$5 \times 5$
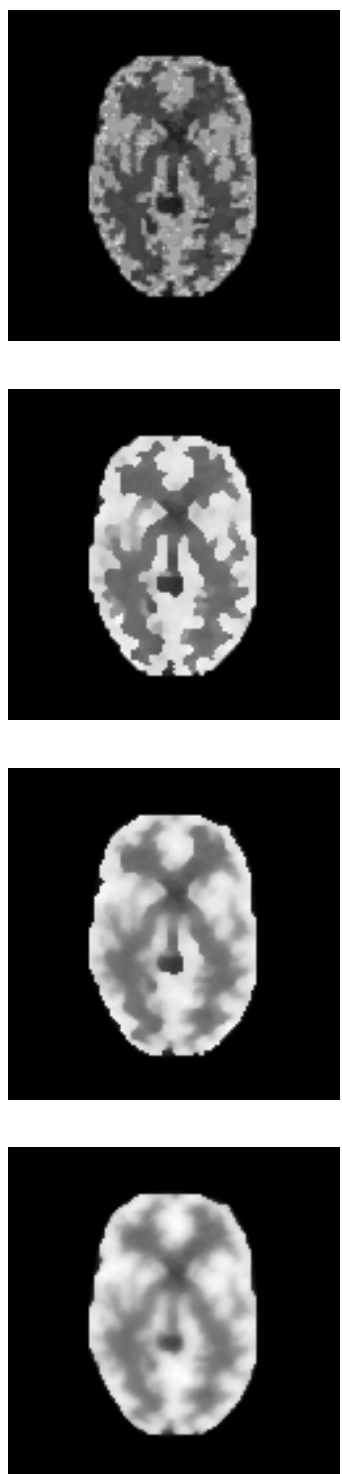

$7 \times 7$
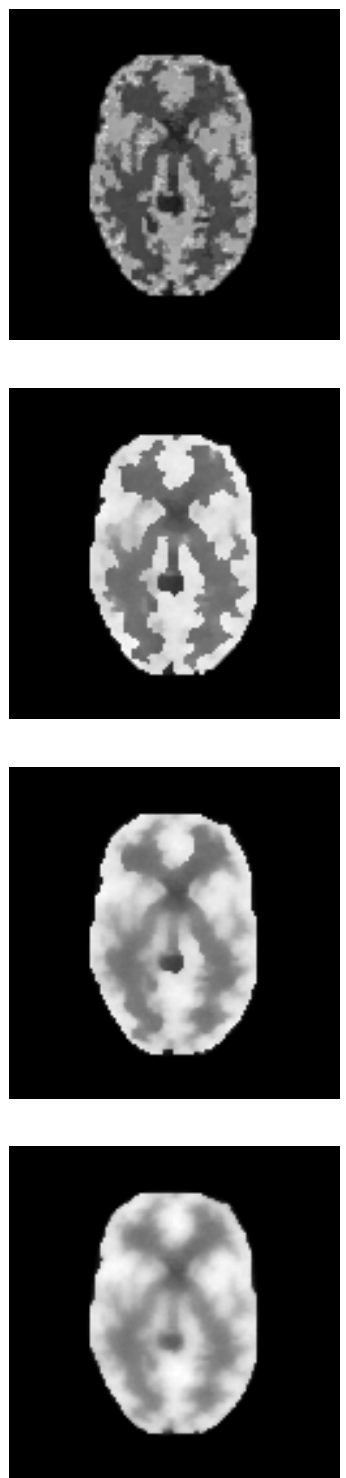

Figure 8: The IIF-MAP Reconstructions with various bilateral filters. ( $\beta=0.25$ for all the case) 

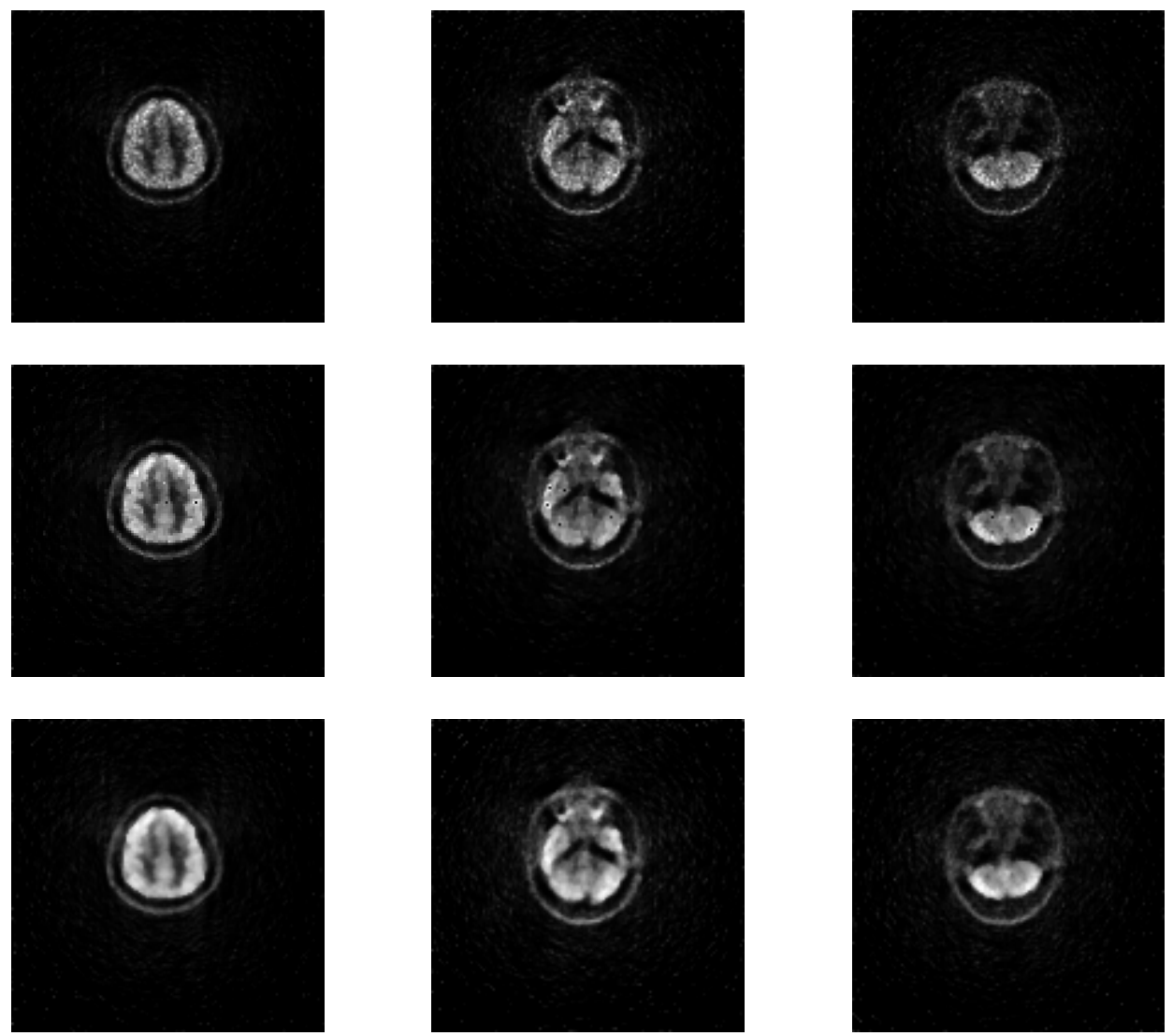

Figure 9: Reconstructions for three different slices using clinical brain PET raw data. From top to bottom: ML-EM, TV-MAP ( $\beta=0.01)$, and IFF-MAP ( $\left.\beta=0.15, \sigma_{R}=0.8\right)$ respectively. 